\title{
Coassociative magmatic bialgebras and the Fine numbers
}

\author{
Ralf Holtkamp · Jean-Louis Loday • María Ronco
}

Received: 4 October 2006 / Accepted: 23 July 2007 / Published online: 25 August 2007

(C) Springer Science+Business Media, LLC 2007

\begin{abstract}
We prove a structure theorem for the connected coassociative magmatic bialgebras. The space of primitive elements is an algebra over an operad called the primitive operad. We prove that the primitive operad is magmatic generated by $n-2$ operations of arity $n$. The dimension of the space of all the $n$-ary operations of this primitive operad turns out to be the Fine number $F_{n-1}$. In short, the triple of operads (As, Mag, MagFine) is good.
\end{abstract}

Keywords Bialgebra $\cdot$ Generalized bialgebra $\cdot$ Hopf algebra .

Cartier-Milnor-Moore · Poincaré-Birkhoff-Witt · Magmatic · Operad · Fine number $\cdot$ Pre-Lie algebra

\section{Introduction}

A magmatic algebra is a vector space equipped with a unital binary operation, denoted $x \cdot y$ with no further assumption. Let us impose that it is also equipped with

The third author work is partially supported by FONDECYT Project 1060224

\footnotetext{
R. Holtkamp ( $\varangle)$

Fakultät für Mathematik, Ruhr-Universität, 44780 Bochum, Germany

e-mail: ralf.holtkamp@ruhr-uni-bochum.de
}

\section{J.-L. Loday}

Institut de Recherche Mathématique Avancée, CNRS et Université Louis Pasteur, 7 rue R. Descartes, 67084 Strasbourg Cedex, France

e-mail: loday@math.u-strasbg.fr

M. Ronco

Departamento de Matematicas, Facultad de Ciencias, Universidad de Valparaiso,

Avda. Gran Bretana 1091, Valparaiso, Chile

e-mail: maria.ronco@uv.cl 
a counital binary cooperation, denoted $\Delta(x)$. There are different compatibility relations that one can suppose between the operation and the cooperation. Here are three of them:

$$
\begin{array}{lrl}
\text { Hopf: } & \Delta(x \cdot y) & =\Delta(x) \cdot \Delta(y) \\
\text { magmatic: } & \Delta(x \cdot y) & =x \cdot y \otimes 1+x \otimes y+1 \otimes x \cdot y \\
& \text { unital infinitesimal: } \Delta(x \cdot y)=\Delta(x) \cdot(1 \otimes y)+(x \otimes 1) \cdot \Delta(y)-x \otimes y
\end{array}
$$

On the free magmatic algebra the cooperation $\Delta$ is completely determined by this choice of compatibility relation and the assumption that the generators are primitive. In the Hopf case $\Delta$ is coassociative and cocommutative, giving rise to the notion of $\mathrm{Com}^{c}$-Mag-bialgebra. This case has been addressed in [9]. In the magmatic case $\Delta$ is comagmatic, giving rise to the notion of $\mathrm{Mag}^{c}$-Mag-bialgebra. This case has been addressed by E. Burgunder in [3]. In the u.i. case, $\Delta$ is coassociative, giving rise to the notion of $A s^{c}$-Mag-bialgebra. This case is the subject of this paper.

First, we construct a functor $F:$ Mag-alg $\rightarrow$ MagFine-alg, where MagFine is the operad of algebras having $(n-2)$ operations of arity $n$ for $n \geq 2$ (no binary operation, one ternary operation, etc.). Then we show that the primitive part of any $A s^{c}$-Magbialgebra is closed under the MagFine operations. The functor $F$ has a left adjoint denoted $U:$ MagFine-alg $\rightarrow$ Mag-alg.

Second, we prove a structure theorem for connected $A s^{c}$-Mag-bialgebras. It says that, as a bialgebra, any connected $A s^{c}$-Mag-bialgebra $\mathcal{H}$ is isomorphic to $U(\operatorname{Prim} \mathcal{H})$ and that, as a coalgebra, it is cofree. Observe that $U(\operatorname{Prim} \mathcal{H})$ has a meaning since the primitive part is a MagFine-algebra. These statements are analogous to the CartierMilnor-Moore theorem [4, 14] and the Poincaré-Birkhoff-Witt theorem for cocommutative (classical) bialgebras.

As a consequence there is an equivalence of categories:

$$
\left\{\text { connected } A s^{c} \text {-Mag-bialg }\right\} \underset{\text { Prim }}{\leftrightarrows}\{\text { MagFine-alg }\}
$$

In the terminology of [10], the triple of operads

$$
\text { (As, Mag, MagFine) }
$$

is a good triple of operads.

As a byproduct of some intermediate lemma we obtain a new combinatorial interpretation of the Fine numbers $F_{n-1}, n=1,2, \ldots$ (cf. Corollary 3.4):

$$
(1,0,1,2,6,18,57,186,622,2120,7338,25724, \ldots) .
$$

In the magmatic case, (Mag, Mag, Vect) is a good triple of operads, see [3].

The study of primitive elements in the Hopf case, cf. [7, 20], leads to a good triple (Com, Mag, Sabinin), due to Shestakov and others. Here the integer series $\frac{1}{(n-1) !} \operatorname{dim} \operatorname{Sabinin}(n)$ is given by the Log-Catalan numbers

$$
(1,1,4,13,46,166,610,2269,8518,32206, \ldots) \text {. }
$$


For other instances of strong relationship between bialgebras and trees (generators of the free magmatic algebra), the reader can look at (nonexhaustive list) $[1,3,8$, 9, 11-13, 19]. At the suggestion of one of the referees, we also give the following references on tree enumeration and combinatorial approaches to the Cartier-MilnorMoore and Poincaré-Birkhoff-Witt theorems: [2, 16-18, 21].

Notation In this paper $\mathbb{K}$ is a field and all vector spaces are over $\mathbb{K}$. Its unit is denoted $1_{\mathbb{K}}$ or just 1 . The category of vector spaces over $\mathbb{K}$ is denoted by Vect. The vector space spanned by the elements of a set $X$ is denoted $\mathbb{K}[X]$. The tensor product of vector spaces over $\mathbb{K}$ is denoted by $\otimes$. The tensor product of $n$ copies of the space $V$ is denoted by $V^{\otimes n}$. For $v_{i} \in V$ the element $v_{1} \otimes \cdots \otimes v_{n}$ of $V^{\otimes n}$ is denoted by $\left(v_{1}, \ldots, v_{n}\right)$ or simply by $v_{1} \ldots v_{n}$. A linear map $V^{\otimes n} \rightarrow V$ is called an $n$-ary operation on $V$ and a linear map $V \rightarrow V^{\otimes n}$ is called an $n$-ary cooperation on $V$. The symmetric group is the automorphism group of the finite set $\{1, \ldots, n\}$ and is denoted $S_{n}$. It acts on $V^{\otimes n}$ by $\sigma \cdot\left(v_{1}, \ldots, v_{n}\right)=\left(v_{\sigma^{-1}(1)}, \ldots, v_{\sigma^{-1}(n)}\right)$. The action is extended to an action of $\mathbb{K}\left[S_{n}\right]$ by linearity.

For a given type of algebras, or algebraic operad $\mathcal{P}$, the category of algebras of type $\mathcal{P}$, also called $\mathcal{P}$-algebras, is denoted $\mathcal{P}$-alg. All the operads $\mathcal{P}$ appearing in this paper are regular operads. So, the space $\mathcal{P}(n)$ of all $n$-ary operations is of the form $\mathcal{P}(n)=\mathcal{P}_{n} \otimes \mathbb{K}\left[S_{n}\right]$. The free algebra of type $\mathcal{P}$ over the vector space $V$ is $\mathcal{P}(V)=\bigoplus_{n \geq 0} \mathcal{P}_{n} \otimes V^{\otimes n}$. We often look at $\mathcal{P}$ as an endofunctor of the category Vect of vector spaces. For more information on algebraic operads see for instance the first section of [10]. For a general textbook on operads, cf. [15].

When $\mathcal{P}=A s$, the operad of unital associative algebras, the free algebra over $V$ is the tensor algebra $T(V)$. We use equivalently the notation $A s(V)$ and $T(V)$.

\section{Coassociative magmatic bialgebras}

In this section we introduce the notion of coassociative magmatic bialgebra and we prove that a free magmatic algebra has a natural structure of $A s^{c}$-Mag-bialgebra. We give an explicit description of the coproduct.

Definition 2.1 A coassociative magmatic bialgebra $\mathcal{H}$, or As ${ }^{c}$-Mag-bialgebra for short, is a vector space $\mathcal{H}$ over $\mathbb{K}$ equipped with a coassociative cooperation $\Delta$ : $\mathcal{H} \rightarrow \mathcal{H} \otimes \mathcal{H}$ and a binary operation $(x, y) \mapsto x \cdot y$, which satisfy the unital infinitesimal relation:

$$
\Delta(x \cdot y)=\Delta(x) \cdot(1 \otimes y)+(x \otimes 1) \cdot \Delta(y)-x \otimes y .
$$

Moreover we suppose that the operation - has a unit 1 , that the cooperation $\Delta$ satisfies the relation $\Delta(1)=1 \otimes 1$, and has a counit $c$ that preserves the units and satisfies $c(x \cdot y)=c(x) c(y)$ for all $x, y \in \mathcal{H}$. The kernel of the augmentation map $\mathcal{H} \rightarrow \mathbb{K}$ is called the augmentation ideal, and is denoted $\overline{\mathcal{H}}$. So there is a direct decomposition $\mathcal{H}=\mathbb{K} 1 \oplus \overline{\mathcal{H}}$. 
Observe that we do not suppose anything else about the operation, in particular it is not supposed to be associative, hence it is a magmatic product.

For any $x \in \mathcal{H}$, we set

$$
\delta(x):=\Delta(x)-x \otimes 1-1 \otimes x .
$$

The induced map $\delta: \overline{\mathcal{H}} \rightarrow \overline{\mathcal{H}} \otimes \overline{\mathcal{H}}$ is called the reduced coproduct.

The compatibility relation satisfied by the reduced coproduct is

$$
\delta(x \cdot y)=\delta(x) \cdot(1 \otimes y)+(x \otimes 1) \cdot \delta(y)+x \otimes y .
$$

or equivalently

$$
\delta(x \cdot y)=x_{(1)} \otimes x_{(2)} \cdot y+\sum x \cdot y_{(1)} \otimes y_{(2)}+x \otimes y,
$$

where $\delta(x)=x_{(1)} \otimes x_{(2)}$ (Sweedler's notation). Here the summation sign is understood. It is called the non-unital infinitesimal compatibility relation and it can be pictured as:

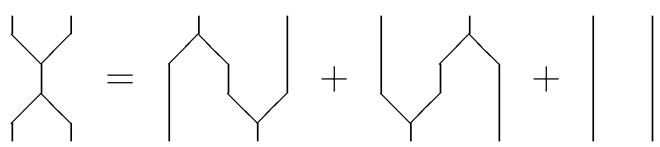

The iterated reduced coproduct $\delta^{n-1}: \overline{\mathcal{H}} \rightarrow \overline{\mathcal{H}}^{\otimes n}$ is defined inductively as $\delta^{1}:=\delta$ and $\delta^{n}:=(\delta \otimes \mathrm{id} \otimes \cdots \otimes \mathrm{id}) \circ \delta^{n-1}$. We say that an $A s^{c}$-Mag-bialgebra $\mathcal{H}$ is connected, or conilpotent, if $\mathcal{H}=\bigcup_{r \geq 0} F_{r} \mathcal{H}$ where $F_{r} \mathcal{H}$ is the coradical filtration of $\mathcal{H}$ defined recursively by the formulas

$$
\begin{aligned}
F_{0} \mathcal{H} & :=\mathbb{K} 1, \\
F_{1} \mathcal{H} & :=\mathbb{K} 1 \oplus \operatorname{Ker} \delta, \\
F_{r} \mathcal{H} & :=\mathbb{K} 1 \oplus\left\{x \in \overline{\mathcal{H}} \mid \delta^{n}(x)=0, \text { for all } n \geq r\right\} .
\end{aligned}
$$

By definition the space of primitive elements is defined as

$$
\operatorname{Prim} \mathcal{H}:=\operatorname{Ker} \delta \subset \overline{\mathcal{H}} .
$$

Free magmatic algebra 2.2 Let us recall that the free magmatic algebra over $V$ is of the form

$$
\operatorname{Mag}(V)=\bigoplus_{n \geq 0} \mathbb{K}\left[P B T_{n}\right] \otimes V^{\otimes n}
$$

where $P B T_{n}$ is the set of planar binary rooted trees with $n$ leaves (and $\mathbb{K}\left[P B T_{0}\right] \otimes$ $V^{\otimes 0}=\mathbb{K} 1$ ). The magmatic product is induced by the grafting of trees and the concatenation of tensors:

$$
\left(t ; v_{1} \ldots v_{p}\right) \cdot\left(s ; v_{p+1} \ldots v_{p+q}\right)=\left(t \vee s ; v_{1} \ldots v_{p+q}\right)
$$

see for instance [8]. 
Proposition 2.3 The free magmatic algebra Mag $(V)$ over the vector space $V$ has a natural structure of coassociative magmatic bialgebra.

Proof The tensor product $\operatorname{Mag}(V) \otimes \operatorname{Mag}(V)$ is equipped with the following magmatic product:

$$
(x \otimes y) \cdot\left(x^{\prime} \otimes y^{\prime}\right):=\left(x \cdot x^{\prime}\right) \otimes\left(y \cdot y^{\prime}\right) .
$$

Since $\operatorname{Mag}(V)$ is free, the map $V \rightarrow \operatorname{Mag}(V) \otimes \operatorname{Mag}(V), v \mapsto v \otimes 1+1 \otimes v$ admits a unique lifting $\Delta: \operatorname{Mag}(V) \rightarrow \operatorname{Mag}(V) \otimes \operatorname{Mag}(V)$ which satisfies the unital infinitesimal relation. Observe that $\Delta$ is not a magmatic homomorphism.

The two ternary cooperations $(\Delta \otimes \mathrm{Id}) \Delta$ and $(\mathrm{Id} \otimes \Delta) \Delta$ agree on $V$ (giving $v \mapsto$ $v \otimes 1 \otimes 1+1 \otimes v \otimes 1+1 \otimes 1 \otimes v$ ). If they agree on $x$ and $y$, then they also agree on $x \cdot y$ by the u.i. relation; in fact they both yield

$$
\begin{aligned}
& (\Delta \otimes \mathrm{Id}) \Delta(x) \cdot(1 \otimes 1 \otimes y)+(\Delta(x) \otimes 1) \cdot(1 \otimes \Delta(y)) \\
& \quad+(x \otimes 1 \otimes 1) \cdot(\Delta \otimes \mathrm{Id}) \Delta(y)-x \otimes \Delta(y)-\Delta(x) \otimes y .
\end{aligned}
$$

Hence they agree on $\operatorname{Mag}(V)$ by induction and so the map $\Delta$ is coassociative.

We denote by Prim $\operatorname{Mag}(V)$ the primitive part of $\operatorname{Mag}(V)$ under this cooperation.

Corollary 2.4 The functor $V \mapsto \operatorname{Prim} \operatorname{Mag}(V)$ is an algebraic operad under the composition of the operad Mag. In other words there is a natural transformation of functors Prim Mag $\circ$ Prim Mag ...> Prim Mag, which makes the following diagram commutative

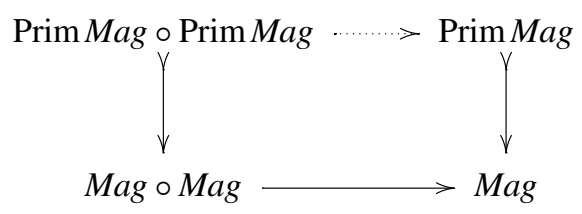

The primitive part of a coassociative magmatic bialgebra is a Prim Mag-algebra.

Proof An $A s^{c}$-Mag-bialgebra is a generalized bialgebra in the sense of [10] because the compatibility relation between the operation and the cooperation is distributive.

In [10] it is proved that, for a generalized bialgebra of type $\mathcal{C}^{c}-\mathcal{A}$, if the free algebra $\mathcal{A}(V)$ is a $\mathcal{C}^{c}-\mathcal{A}$-bialgebra, then $\operatorname{Prim} \mathcal{A}(V)$ determines an operad. By Proposition 2.3 this hypothesis is fulfilled in the $A s^{c}$-Mag-case, whence the assertion.

The coproduct $\delta$ made explicit 2.5 Let $\mathcal{H}$ be an $A s^{c}$-Mag-bialgebra over the field $\mathbb{K}$. Let $\omega^{n}$ be the operation which corresponds to the left comb, that is

$$
\omega^{n}\left(x_{1}, \ldots, x_{n}\right):=\left(\left(\ldots\left(\left(x_{1} \cdot x_{2}\right) \cdot x_{3}\right) \ldots\right) \cdot x_{n}\right) .
$$


Given primitive elements $x_{1}, \ldots, x_{n}$ of $\mathcal{H}$, it is immediate to check that:

$$
\begin{aligned}
\delta\left(\omega^{n}\left(x_{1}, \ldots, x_{n}\right)\right)= & x_{1} \otimes \omega^{n-1}\left(x_{2}, \ldots, x_{n}\right)+\cdots \\
& +\omega^{i}\left(x_{1}, \ldots, x_{i}\right) \otimes \omega^{n-i}\left(x_{i+1}, \ldots, x_{n}\right) \\
& +\cdots+\omega^{n-1}\left(x_{1}, \ldots, x_{n-1}\right) \otimes x_{n} .
\end{aligned}
$$

By Proposition 2.3, the free magmatic algebra is a coassociative magmatic bialgebra, where the product is induced by the grafting of trees.

We now give an explicit description of the homomorphism $\delta$, defined in Proposition 2.3 , in terms of splitting of trees. Let $t$ be a planar binary rooted tree with $r$ leaves numbered from left to right by $1,2, \ldots, r$. Let $i, 1 \leq i<r$, be an integer. We split the tree $t$ into two trees $t_{(1)}^{i}$ and $t_{(2)}^{i}$ by cutting in between the leaves $i$ and $i+1$. More precisely, the tree $t_{(1)}^{i}$ is the part of $t$ which is on the left side of the path from leaf $i$ to the root (including the path). The tree $t_{(2)}^{i}$ is the analogous part on the right side of the path which runs from leaf $i+1$ to the root. For instance,

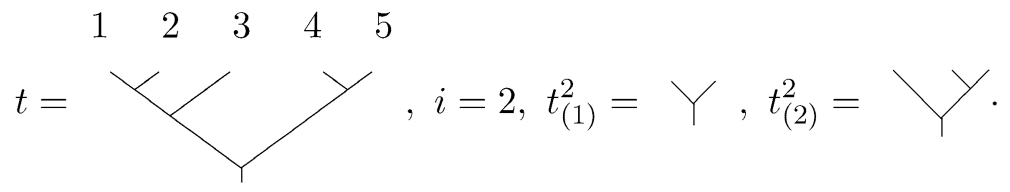

Lemma 2.6 Let Mag $(V)$ be the free magmatic algebra on $V$ equipped with its coassociative magmatic bialgebra structure. Then the linear map $\delta$ applied to an element $\left(t ; v_{1} \ldots v_{r}\right)$ can be written as a sum $\sum_{i=1}^{r-1} \delta_{i}$, where

$$
\delta_{i}\left(t ; v_{1} \ldots v_{r}\right)=\left(t_{(1)}^{i} ; v_{1} \ldots v_{i}\right) \otimes\left(t_{(2)}^{i} ; v_{i+1} \ldots v_{r}\right)
$$

Proof The result is immediate for $r=1,2$. For $r>2$, there exist unique trees $t^{l}$ and $t^{r}$ such that $t=t^{l} \vee t^{r}$, with $t^{l} \in P B T_{k}$ and $t^{r} \in P B T_{r-k}$ for some $1 \leq k \leq r-1$. Applying the recursive hypothesis and the compatibility relation between the product - and the coproduct $\delta$ (nonunital infinitesimal relation), one gets:

$$
\begin{aligned}
& \delta\left(t ; v_{1} \ldots v_{r}\right)=\delta\left(\left(t^{l} ; v_{1} \ldots v_{k}\right) \cdot\left(t^{r} ; v_{k+1} \ldots v_{r}\right)\right)= \\
& \quad \sum_{i=1}^{k-1}\left(\left(t^{l}\right)_{(1)}^{i} ; v_{1} \ldots v_{i}\right) \otimes\left(\left(t^{l}\right)_{(2)}^{i} \vee t^{r} ; v_{i+1} \ldots v_{r}\right) \\
& \quad+\left(t^{l} ; v_{1} \ldots v_{k}\right) \otimes\left(t^{r} ; v_{k+1} \ldots v_{r}\right) \\
& \quad+\sum_{i=k+1}^{r-1}\left(t^{l} \vee\left(t^{r}\right)_{(1)}^{i-k} ; v_{1} \ldots v_{i}\right) \otimes\left(\left(t^{r}\right)_{(2)}^{i-k} ; v_{i+1} \ldots v_{r}\right) .
\end{aligned}
$$

This formula implies that $\sum_{i=1}^{r-1} \delta_{i}$ satisfies the n.u.i. compatibility relation, therefore it is the reduced coproduct of Proposition 2.3. 


\section{MagFine algebras}

We introduce the notion of MagFine-algebra and we compute dim MagFine ${ }_{n}$. We mention a new combinatorial interpretation of the Fine numbers. We construct a functor Mag-alg $\rightarrow$ MagFine-alg. We will later show that it factors surjectively through Prim Mag-alg.

MagFine algebras 3.1 By definition a MagFine algebra is a vector space endowed with $(n-2) n$-ary operations denoted $m_{i}^{n}, 1 \leq i \leq n-2$, for all $n \geq 3$. Let MagFine be the operad associated to this type of algebras. Since the generating operations are not supposed to satisfy any symmetry property nor any relations, the operad is regular. In particular the space of $n$-ary operations is of the form

$$
\operatorname{MagFine}(n)=\text { MagFine }_{n} \otimes \mathbb{K}\left[S_{n}\right] \text {. }
$$

Proposition 3.2 The dimension of MagFine ${ }_{n}$ is the Fine number $F_{n-1}$ defined by the generating series

$$
F(x)=\sum_{n \geq 1} F_{n-1} x^{n}=\frac{1+2 x-\sqrt{1-4 x}}{2(2+x)} .
$$

Proof Let $\mathcal{P}$ be the operad defined by $(n-2) n$-ary operations and whose relations are: any nontrivial composition is zero. This is a nilpotent operad, so its Koszul dual is free with the same generators. Hence the Koszul dual operad of $\mathcal{P}$ is $\mathcal{P}^{!}=$MagFine.

We will use a result of B. Vallette [22], based on the Koszul duality theory of operads (cf. [6]), which relates the dimensions of $\mathcal{P}(n)$ and of $\mathcal{P}^{!}(n)$.

For a presented quadratic operad $\mathcal{P}$ we let $\mathcal{P}^{(d)}(n)$ be the space spanned by the $n$-ary operations constructed out of $d$ generating operations. In the case at hand we have $\operatorname{dim} \mathcal{P}^{(0)}(1)=1, \operatorname{dim} \mathcal{P}^{(1)}(n)=(n-2) n !$ and $\operatorname{dim} \mathcal{P}^{(d)}(n)=0$ otherwise.

It is proved in [22] Section 9, that the Poincaré series

$$
f_{\mathcal{P}}(x, z):=\sum_{n \geq 1, d \geq 0} \frac{\operatorname{dim} \mathcal{P}^{(d)}(n)}{n !} x^{n} z^{d}
$$

of $\mathcal{P}$ and $f_{\mathcal{P} !}(x, z)$ are related by the relation

$$
f_{\mathcal{P} !}\left(f_{\mathcal{P}}(x, z),-z\right)=x,
$$

when $\mathcal{P}$ is a Koszul operad. Since $\mathcal{P}$ ! is a free operad, it is Koszul (cf. [6, 22]). Thus we can apply this formula, to get the desired enumeration result (cf. [2] for related enumeration techniques via Hilbert series and species).

We know that, in our case,

$$
f_{\mathcal{P}}(x, z)=x+z \sum_{n \geq 3}(n-2) x^{n}=x+z \frac{x^{3}}{(1-x)^{2}} .
$$


We want to compute the numbers $\operatorname{dim}$ MagFine $_{n}$, or equivalently the series

$$
F(x)=\sum_{n \geq 1} \operatorname{dim} \text { MagFine }_{n} x^{n}=\sum_{n \geq 1} \frac{\operatorname{dim} \operatorname{MagFine}(n)}{n !} x^{n}=f_{\mathcal{P} !}(x, 1) .
$$

Applying Vallette's formula for $z=-1$ we get

$$
F\left(x-\frac{x^{3}}{(1-x)^{2}}\right)=x .
$$

It follows that $F(x)=\frac{1+2 x-\sqrt{1-4 x}}{2(2+x)}$. This is exactly the generating function of the Fine numbers, cf. [5].

Remark 3.3 The Fine numbers are, in low dimension (starting at $F_{0}=1$ ),

$$
1,0,1,2,6,18,57,186,622,2120,7338,25724, \ldots .
$$

They admit several combinatorial interpretations, cf. [5]. Here is a new one, which is a consequence of Proposition 3.2 (but which can be proved by combinatorial arguments).

Corollary 3.4 Let $X_{n}$ be the set of planar rooted trees with $n$ leaves, with no unary nor binary vertices and whose $k$-ary vertices are labelled by $\{1, \ldots, k-2\}$. Then the following equality holds: $\# X_{n}=F_{n-1}$ (Fine number).

Proof It is immediate to check that the trees in $X_{n}$ are in bijection with a linear basis of MagFine ${ }_{n}$.

Higher operations on a magmatic algebra 3.5 The associator operation as is a ternary operation defined as

$$
\operatorname{as}\left(x_{1}, x_{2}, x_{3}\right):=\left(x_{1} \cdot x_{2}\right) \cdot x_{3}-x_{1} \cdot\left(x_{2} \cdot x_{3}\right) .
$$

If $(A, \cdot)$ is any magmatic algebra over $\mathbb{K}$, we can equip it with the $n$-ary operations $\mu_{i}^{n}: A^{\otimes n} \rightarrow A$, for all $n \geq 3$, all $1 \leq i \leq n-2$, defined as follows:

$$
\begin{aligned}
\mu_{1}^{3}\left(x_{1}, \ldots, x_{3}\right) & :=\operatorname{as}\left(x_{1}, x_{2}, x_{3}\right), \\
\mu_{1}^{n}\left(x_{1}, \ldots, x_{n}\right) & :=\operatorname{as}\left(x_{1}, \omega^{n-2}\left(x_{2}, \ldots, x_{n-1}\right), x_{n}\right), \\
\mu_{2}^{4}\left(x_{1}, \ldots, x_{4}\right) & :=\operatorname{as}\left(x_{1}, x_{2}, x_{3} \cdot x_{4}\right)-\operatorname{as}\left(x_{1}, x_{2}, x_{3}\right) \cdot x_{4} \\
\mu_{i}^{n}\left(x_{1}, \ldots, x_{n}\right) & :=\mu_{2}^{4}\left(x_{1}, \omega^{n-i-1}\left(x_{2}, \ldots, x_{n-i}\right), \omega^{i-1}\left(x_{n-i+1}, \ldots, x_{n-1}\right), x_{n}\right),
\end{aligned}
$$

for $x_{1}, \ldots, x_{n} \in A, n \geq 4$, and $2 \leq i \leq n-2$. We recall that $\omega^{n}$ is the left comb product.

In other words we have constructed a morphism of operads

$$
\text { MagFine } \rightarrow \text { Mag, } \quad m_{i}^{n} \mapsto \mu_{i}^{n} .
$$


In the following, we show that its image is contained in Prim Mag.

Associator 3.6 The associator operation as is a Prim Mag-operation: as $\in$ Prim Mag (3). In other words, for any $A s^{c}$-Mag-bialgebra $\mathcal{H}$, and primitive elements $x_{1}, x_{2}, x_{3}$, the element $a s\left(x_{1}, x_{2}, x_{3}\right)$ is again primitive. This is a special case of the following lemma:

Lemma 3.7 Let $\mathcal{H}$ be an As $s^{c}$-Mag-bialgebra, and let $x \in \operatorname{Prim} \mathcal{H}, y, z \in \mathcal{H}$. Then:

$$
\delta(a s(x, y, z))=\sum a s\left(x, y, z_{(1)}\right) \otimes z_{(2)} .
$$

Proof We use the compatibility relation given in Definition 2.1. Since $x \in \operatorname{Prim} \mathcal{H}$, $\delta(x \cdot y)=(x \otimes 1) \cdot \delta(y)+x \otimes y=\sum x \cdot y_{(1)} \otimes y_{(2)}+x \otimes y$, and $\delta(\operatorname{as}(x, y, z))$ is given by

$$
\begin{aligned}
& \sum x \cdot y_{(1)} \otimes y_{(2)} \cdot z+x \otimes y \cdot z+\sum(x \cdot y) \cdot z_{(1)} \otimes z_{(2)}+x \cdot y \otimes z \\
& \quad-\sum x \cdot y_{(1)} \otimes y_{(2)} \cdot z-\sum x \cdot\left(y \cdot z_{(1)}\right) \otimes z_{(2)}-x \cdot y \otimes z-x \otimes y \cdot z \\
& =\sum a s\left(x, y, z_{(1)}\right) \otimes z_{(2)} .
\end{aligned}
$$

Proposition 3.8 If $\mathcal{H}$ is an $A s^{c}$-Mag-bialgebra, then Prim $\mathcal{H}$ is closed under the operations $\mu_{i}^{n}$, for $n \geq 3$ and $1 \leq i \leq n-2$ (in other words the operations $\mu_{i}^{n}$ lie in $\operatorname{Prim} \operatorname{Mag}(n))$.

Proof For all $n \geq 3,2 \leq i \leq n-2$, and $x_{1}, \ldots, x_{n} \in \operatorname{Prim} \mathcal{H}$, we have to show that $\mu_{i}^{n}\left(x_{1}, \ldots, x_{n}\right) \in \operatorname{Prim} \mathcal{H}$, too.

Using Lemma 3.7 in the case where $z=x_{n} \in \operatorname{Prim} \mathcal{H}$, we immediately get $\mu_{1}^{n}\left(x_{1}, \ldots, x_{n}\right) \in \operatorname{Prim} \mathcal{H}$ for all $n$.

We need to compute $\delta\left(\mu_{i}^{n}\left(x_{1}, \ldots, x_{n}\right)\right)$, which is, in short notation,

$$
\delta\left(\operatorname{as}\left(x_{1}, \omega^{n-i-1}, \omega^{i}\right)\right)-\delta\left(\operatorname{as}\left(x_{1}, \omega^{n-i-1}, \omega^{i-1}\right) \cdot x_{n}\right) .
$$

By Lemma 3.7 (together with 2.5) it follows that

$$
\delta\left(\operatorname{as}\left(x_{1}, \omega^{n-i-1}, \omega^{i}\right)\right)=\sum_{j=1}^{i-1} a s\left(x_{1}, \omega^{n-i-1}, \omega^{i-j}\right) \otimes \omega^{j} .
$$

Similarly,

$$
\begin{aligned}
\delta & \left(\operatorname{as}\left(x_{1}, \omega^{n-i-1}, \omega^{i-1}\right) \cdot x_{n}\right) \\
& =\sum_{j=1}^{i-2} a s\left(x_{1}, \omega^{n-i-1}, \omega^{i-j}\right) \otimes \omega^{j} \cdot x_{n}+a s\left(x_{1}, \omega^{n-i-1}, \omega^{i-1}\right) \otimes x_{n} \\
& =\sum_{j=1}^{i-1} \operatorname{as}\left(x_{1}, \omega^{n-i-1}, \omega^{i-j}\right) \otimes \omega^{j}
\end{aligned}
$$


which ends the proof.

From MagFine to Mag 3.9 Proposition 3.8 provides us with a map $\theta$ :MagFine( $V)$

$\rightarrow \operatorname{Prim} \operatorname{Mag}(V)$ by sending $m_{i}^{n}$ to $\mu_{i}^{n}$.

We extend it to a map $\theta: T(\operatorname{MagFine}(V)) \rightarrow \operatorname{Mag}(V)$ as the composite

$$
T(\operatorname{MagFine}(V)) \rightarrow T(\operatorname{Prim} \operatorname{Mag}(V)) \stackrel{\omega^{\bullet}}{\rightarrow} \operatorname{Mag}(V)
$$

So we have $\theta\left(y_{1}, \ldots, y_{r}\right)=\omega^{r}\left(\theta\left(y_{1}\right), \ldots, \theta\left(y_{r}\right)\right)$ for $y_{i} \in \operatorname{MagFine}(V)$.

We want to prove the following result.

Proposition 3.10 The map

$$
\theta: T(\operatorname{MagFine}(V)) \rightarrow \operatorname{Mag}(V)
$$

is surjective.

Let $P \operatorname{Mag}(V)$ be the smallest subspace of $\operatorname{Mag}(V)$ which contains $V$ and is closed under all operations $\mu_{i}^{n}$. All elements of $\operatorname{PMag}(V)$ are primitive, by Proposition 3.8. They can be presented by (linear combinations of) compositions of operations $\mu_{i}^{n}$, evaluated on elements of $V$. Let $\Omega(P \operatorname{Mag}(V))$ be the subspace of $\operatorname{Mag}(V)$ spanned by the elements of type $\omega^{r}\left(z_{1}, \ldots, z_{r}\right)$, with $r \geq 1$ and $z_{i} \in P \operatorname{Mag}(V)$, for $1 \leq i \leq r$.

We will show that the subspace $\Omega(P \operatorname{Mag}(V))$ coincides with the whole space $\operatorname{Mag}(V)$.

We need the following two lemmas.

Lemma 3.11 Let $z, u_{1}, \ldots, u_{k}$ be elements of $P \operatorname{Mag}(V)$. The element $z \cdot \omega^{k}\left(u_{1}\right.$, $\left.\ldots, u_{k}\right)$ belongs to $\Omega(P \operatorname{Mag}(V))$.

Proof For $k=1$ the result is obviously true.

For $k \geq 2$, we have that:

$$
z \cdot \omega^{k}\left(u_{1}, \ldots, u_{k}\right)=-\mu_{1}^{k+1}\left(z, u_{1}, \ldots, u_{k}\right)+\left(z \cdot \omega^{k-1}\left(u_{1}, \ldots, u_{k-1}\right)\right) \cdot u_{k} .
$$

By the recursive hypothesis on $k$, the element $z \cdot \omega^{k-1}\left(u_{1}, \ldots, u_{k-1}\right)$ belongs to $\Omega(P \operatorname{Mag}(V))$. Since $-\mu_{1}^{k+1}\left(z, u_{1}, \ldots, u_{k}\right)$ belongs to $\operatorname{PMag}(V)$, we get: $z \cdot \omega^{k}\left(u_{1}, \ldots, u_{k}\right) \in \Omega(P \operatorname{Mag}(V))$.

Lemma 3.12 Let $z_{1}, \ldots, z_{r}$ be elements of PMag( $\left.V\right)$. The element $\omega^{r}\left(z_{1}, \ldots, z_{r}\right)$ belongs to $P \operatorname{Mag}(V) \cdot(\mathbb{K} \oplus \Omega(P \operatorname{Mag}(V)))$.

Proof The result is immediate for $r=1,2$. For $r \geq 3$, note that:

$$
\begin{aligned}
\omega^{r}\left(z_{1}, \ldots, z_{r}\right)= & \omega^{r-2}\left(\mu_{1}^{3}\left(z_{1}, z_{2}, z_{3}\right), z_{4}, \ldots, z_{r}\right) \\
& +\omega^{r-3}\left(\mu_{1}^{4}\left(z_{1}, \ldots, z_{4}\right), z_{5}, \ldots, z_{r}\right) \\
& +\cdots+\mu_{1}^{r}\left(z_{1}, \ldots, z_{r}\right)+z_{1} \cdot \omega^{r-1}\left(z_{2}, \ldots, z_{r}\right) .
\end{aligned}
$$


The result follows immediately using the recursive hypothesis and the equality above.

Proof (Continuation of the Proof of Proposition 3.10) In degrees smaller than or equal to two, the result is immediate.

It suffices to check the result on any homogeneous element $x=x_{1} \cdot x_{2}$, with $x_{1}$ and $x_{2}$ homogeneous elements, both of degree smaller than $\operatorname{deg}(x)$.

Applying the recursive hypothesis, we restrict ourselves to prove that any element of the form $\omega^{r}\left(z_{1}, \ldots, z_{r}\right) \cdot \omega^{k}\left(u_{1}, \ldots, u_{k}\right)$, with $z_{1}, \ldots, z_{r}, u_{1}, \ldots, u_{k}$ in $\operatorname{PMag}(V)$ belongs to $\Omega(P \operatorname{Mag}(V))$.

By Lemma 3.12, the element $\omega^{r}\left(z_{1}, \ldots, z_{r}\right)$ is in $\operatorname{PMag}(V) \cdot(\mathbb{K} \oplus \Omega(P \operatorname{Mag}(V)))$. So, it suffices to prove the assertion for any element $x=\left(z_{1} \cdot \omega^{r-1}\left(z_{1}, \ldots, z_{r}\right)\right)$. $\omega^{k}\left(u_{1}, \ldots, u_{k}\right)$, with $r, k \geq 1$ and the elements $z_{1}, \ldots, z_{r}, u_{1}, \ldots, u_{k}$ in PMag $(V)$.

Applying the formula of $\mu_{k}^{r+k}\left(z_{1}, \ldots, z_{r}, u_{1}, \ldots, u_{k}\right)$, we get:

$$
\begin{aligned}
& \left(z_{1} \cdot \omega^{r-1}\left(z_{1}, \ldots, z_{r}\right)\right) \cdot \omega^{k}\left(u_{1}, \ldots, u_{k}\right) \\
& =\mu_{k}^{r+k}\left(z_{1}, \ldots, z_{r}, u_{1}, \ldots, u_{k}\right)+z_{1} \cdot\left(\omega^{r-1}\left(z_{2}, \ldots, z_{r}\right) \cdot \omega^{k}\left(u_{1}, \ldots, u_{k}\right)\right) \\
& \quad+\operatorname{as}\left(z_{1}, \omega^{r-1}\left(z_{2}, \ldots, z_{r}\right), \omega^{k-1}\left(u_{1}, \ldots, u_{k-1}\right)\right) \cdot u_{k} .
\end{aligned}
$$

By Lemma 3.11, we have that $z_{1} \cdot\left(\omega^{r-1}\left(z_{2}, \ldots, z_{r}\right) \cdot \omega^{k}\left(u_{1}, \ldots, u_{k}\right)\right) \in$ $\Omega(P \operatorname{Mag}(V))$. By the recursive hypothesis on the degree of $x$, the element

$$
\operatorname{as}\left(z_{1}, \omega^{r-1}\left(z_{2}, \ldots, z_{r}\right), \omega^{k-1}\left(u_{1}, \ldots, u_{k-1}\right)\right)
$$

is in $\Omega(P \operatorname{Mag}(V))$, which implies that the element

$$
\operatorname{as}\left(z_{1}, \omega^{r-1}\left(z_{2}, \ldots, z_{r}\right), \omega^{k-1}\left(u_{1}, \ldots, u_{k-1}\right)\right) \cdot u_{k}
$$

belongs to $\Omega(P \operatorname{Mag}(V))$, too.

So, $\left(z_{1} \cdot \omega^{r-1}\left(z_{1}, \ldots, z_{r}\right)\right) \cdot \omega^{k}\left(u_{1}, \ldots, u_{k}\right)$ belongs to $\Omega(P \operatorname{Mag}(V))$ as expected.

The Remark above and Proposition 3.10 imply the following result.

Corollary 3.13 Given a vector space $V$, the morphism $\theta: \operatorname{MagFine}(V) \longrightarrow$ $\operatorname{Prim} \operatorname{Mag}(V)$ is surjective. In particular, the morphism of operads MagFine $\longrightarrow$ Prim Mag is surjective.

Proof We know that $P \operatorname{Mag}(V) \subseteq \operatorname{Prim} \operatorname{Mag}(V)$, and we will check that these spaces are equal. Let $x$ be an element in Prim $\operatorname{Mag}(V)$. By Proposition 3.10 we know that $x=\sum_{i=1}^{n} x^{i}$, where $x^{i}=\sum \omega^{i}\left(x_{1}^{i}, \ldots, x_{i}^{i}\right)$ for some integer $n$ and some elements $x_{j}^{i} \in P \operatorname{Mag}(V)$ for all $i, j$. We know that for any primitive elements $y_{1}, \ldots, y_{n}$ in an $A s^{c}$-Mag-bialgebra we have:

$$
\delta^{r}\left(\omega^{n}\left(y_{1}, \ldots, y_{n}\right)\right)= \begin{cases}0, & \text { for } r \geq n \\ y_{1} \otimes \cdots \otimes y_{n}, & \text { for } r=n-1\end{cases}
$$


Therefore, since $x$ is primitive, we get, for $n \geq 2$,

$$
0=\delta^{n-1}(x)=\sum x_{1}^{n} \otimes \cdots \otimes x_{n}^{n},
$$

which implies $x^{n}=0$ whenever $n \geq 2$. Therefore we get $x=x^{1} \in P \operatorname{Mag}(V)$ and so $\operatorname{PMag}(V)=\operatorname{Prim} \operatorname{Mag}(V)$.

\section{On the structure of coassociative magmatic bialgebras}

We constructed a functor

$$
F: \text { Mag-alg } \rightarrow \text { MagFine-alg, } F(A, \cdot)=\left(A, \mu_{i}^{n}\right) .
$$

We denote by $U:$ MagFine-alg $\rightarrow$ Mag-alg the left adjoint functor of $F$. So, if $\mathrm{A}$ is a MagFine-algebra, then $U(A)$ is the quotient of $\operatorname{Mag}(A)$ by the relations which consist of identifying the operations $m_{i}^{n}$ on $A$ and the operations $\mu_{i}^{n}$ on $\operatorname{Mag}(A)$ which are deduced from the magmatic operation $(x, y) \mapsto x \cdot y$.

The primitive part of a coassociative magmatic bialgebra is a Prim Mag-algebra by Proposition 2.3 and therefore it is a MagFine algebra by Proposition 3.8. Hence we can apply the functor $U$ to $\operatorname{Prim}(\mathcal{H})$ for any $A s^{c}$-Mag-bialgebra $\mathcal{H}$.

The second aim of this paper is to prove the structure theorem for the triple of operads (As, Mag, MagFine).

Theorem 4.1 Let $\mathcal{H}$ be an As ${ }^{c}$-Mag-bialgebra over the field $\mathbb{K}$. Then, TFAE:

(a) $\mathcal{H}$ is connected (or conilpotent),

(b) $\mathcal{H}$ is isomorphic to $U(\operatorname{Prim} \mathcal{H})$,

(c) $\mathcal{H}$ is cofree in the category of connected coassociative coalgebras.

In other words, (As, Mag, MagFine) is a good triple of operads in the sense of [10].

Observe that we did not make any characteristic assumption on $\mathbb{K}$.

Proof The proof of this theorem is in two steps. First, we apply a result of [10] to show that the structure theorem holds for the triple of operads (As, Mag, Prim Mag), cf. Proposition 4.2. Second, we show that the morphism of operads MagFine $\rightarrow$ Prim Mag is an isomorphism, cf. Proposition 4.3.

In the Appendix we give an alternative proof which is based on the construction of an explicit functorial projection $\mathcal{H} \rightarrow \operatorname{Prim} \mathcal{H}$.

Proposition 4.2 The structure theorem holds for the triple

$$
\text { (As, Mag, Prim Mag). }
$$

Proof In [10] it is proved that for any $\mathcal{C}^{c}-\mathcal{A}$-bialgebra type whose compatibility is distributive and which satisfies the hypotheses $(\mathrm{H} 1)$ and $(\mathrm{H} 2)$ recalled below, the structure theorem holds. 
The condition (H0) says that the generating operation and the generating cooperation satisfy a distributive compatibility relation. This condition is checked by direct inspection, see Definition 2.1.

The condition (H1) is the following: the free $\mathcal{A}$-algebra is equipped with a natural structure of $\mathcal{C}^{c}-\mathcal{A}$-bialgebra.

In Proposition 2.3 we proved that $\operatorname{Mag}(V)$ is a $A s^{c}$-Mag-bialgebra.

The condition (H2) is the following: the coalgebra map $\mathcal{A}(V) \rightarrow \mathcal{C}^{c}(V)$ has a natural splitting as coalgebra map.

Let us construct a coalgebra splitting of the map $\operatorname{Mag}(V) \rightarrow A s^{c}(V)$. We simply send the generator $\mu_{n}^{c} \in A s_{n}^{c}$ to the left comb $\omega^{n} \in M a g_{n}$. Lemma 2.6 shows that it induces a coalgebra map $A s^{c}(V) \rightarrow \operatorname{Mag}(V)$.

Proposition 4.3 The morphism of operads MagFine $\rightarrow$ Prim Mag constructed in Section 2 is an isomorphism.

In particular, there is an isomorphism of functors

$$
\operatorname{Mag}=A s^{c} \circ \operatorname{Prim} M a g, \text { i.e. } \operatorname{Mag}(V)=T(\operatorname{Prim} \operatorname{Mag}(V)) .
$$

Proof By Proposition 3.10 and Proposition 4.2 the maps $\theta_{n}:$ MagFine $_{n} \rightarrow$ Prim Mag $_{n}$ are surjective. So it is sufficient to prove that the spaces MagFine ${ }_{n}$ and Prim Mag have the same dimension. In 3.2 we showed that $\operatorname{dim}$ MagFine ${ }_{n}=F_{n-1}$ (Fine number). Let us show that $\operatorname{dim}$ Prim $\mathrm{Mag}_{n}=F_{n-1}$.

Recall that the Poincaré series of a regular operad $\mathcal{P}$ is $f_{\mathcal{P}}(x):=\sum_{n \geq 1} \operatorname{dim} \mathcal{P}_{n} x^{n}$.

By Proposition 4.2, we have an isomorphism

$$
\text { Mag }=A s^{c} \circ \operatorname{Prim} \text { Mag. }
$$

From this isomorphism it follows that the Poincare series of these functors are related by

$$
f_{\text {Mag }}(x)=f_{\text {As }}\left(f_{\text {Prim Mag }}(x)\right) .
$$

Since $f_{\text {Mag }}(x)=\frac{1-\sqrt{1-4 x}}{2}$ and $f_{A s}(x)=\frac{x}{1-x}$, we get

$$
f_{\text {Prim Mag }}(x)=\frac{1+2 x-\sqrt{1-4 x}}{2(2+x)} .
$$

Since this is precisely the generating series of the Fine numbers (cf. [5]), as recalled in 3.2, we have $\operatorname{dim} \operatorname{Prim} \operatorname{Mag}_{n}=F_{n-1}$.

Corollary 4.4 There is an equivalence of categories:

$$
\left\{\text { con. As } s^{c} \text {-Mag-bialg }\right\} \underset{\text { Prim }}{\leftrightarrows}\{\text { MagFine-alg }\}
$$

Corollary 4.5 There is an isomorphism of functors

$$
\text { Mag }=\text { As } \circ \text { MagFine. }
$$


Good triples of operads 4.6 In the terminology of [10] the triple of operads (As, Mag, MagFine) is good, which means that the structure theorem holds. The interesting point is that it determines many other good triples, since, by a result of [10], it suffices to take some relation in MagFine to construct a new good triple. For instance, if we mod out by the associator, then obviously the quotient of MagFine becomes Vect since all the higher operations are constructed from the associator. The middle operad becomes $A s$ and we get the triple $(A s, A s, V e c t)$ which has already been shown to be good in [13].

If we mod out by the pre-Lie relation

$$
\operatorname{as}(x, y, z)=\operatorname{as}(x, z, y)
$$

then the middle operad becomes the operad preLie of pre-Lie algebras, and we get a good triple of operads

$$
\text { (As, preLie, MagFine } \cong \text { ). }
$$

From the isomorphism of functors preLie $=A s^{c} \circ($ MagFine $/ \cong)$ one can compute the dimension of (MagFine/ $\cong$ ) (n) from $n=1$ to $n=5$ :

$$
1,0,3,16,165 \text {. }
$$

It would be nice to find a small presentation of the operad MagFine/ $\cong$.

\section{Appendix}

Self-contained proof of the structure theorem for (As, Mag, Prim Mag) using an explicit idempotent

We first construct an explicit idempotent and we show some of its properties. Then we give a proof of the structure theorem. The use of idempotents for the classical structure theorems (which we do not need here) goes back to Solomon [21].

The idempotent 5.1 Let $\mathcal{H}$ be a connected $A s^{c}$-Mag-bialgebra over the field $\mathbb{K}$. We define an operator $e: \overline{\mathcal{H}} \rightarrow \overline{\mathcal{H}}$ as follows:

$$
e=\mathrm{id}+\sum_{n \geq 1}(-1)^{n} \omega^{n+1} \circ \delta^{n}
$$

where $\omega^{n}$ is the left comb product (cf. 2.5) and $\delta^{n}$ is the iterated reduced coproduct (cf. 2.1). Note that the connectedness of $\mathcal{H}$ implies that $e$ is well-defined.

For any family of elements $x_{1}, \ldots, x_{n}$ in $\mathcal{H}$, let $\overrightarrow{\omega^{n}}\left(x_{1}, \ldots x_{n}\right)$ be the right comb:

$$
\overrightarrow{\omega^{n}}\left(x_{1}, \ldots x_{n}\right):=x_{1} \cdot\left(x_{2} \cdot\left(\ldots \cdot\left(x_{n-1} \cdot x_{n}\right) \ldots\right)\right) .
$$

Lemma 5.2 The operator $e: \overline{\mathcal{H}} \rightarrow \overline{\mathcal{H}}$ has the following properties:

(i) $e\left(\overrightarrow{\omega^{n}}\left(x_{1}, \ldots, x_{n}\right)\right)=0$ for all $x_{1}, \ldots, x_{n} \in \operatorname{Prim} \mathcal{H}$. 
(ii) $\delta \circ e=0$.

(iii) $\left.e\right|_{\operatorname{Prim} \mathcal{H}}=\mathrm{id}_{\operatorname{Prim}} \mathcal{H}$.

Proof (i) Note that if the elements $x_{1}, \ldots, x_{n}$ belong to $\operatorname{Prim}(\mathcal{H})$, then:

$$
\delta^{k}\left(\overrightarrow{\omega^{n}}\left(x_{1}, \ldots, x_{n}\right)\right)=\sum_{1 \leq i_{1}<\ldots<i_{k} \leq n} \overrightarrow{\omega^{i_{1}}}\left(x_{1}, \ldots, x_{i_{1}}\right) \otimes \cdots \otimes \overrightarrow{\omega^{n-l_{k}}}\left(x_{i_{k}+1}, \ldots, x_{n}\right) .
$$

So, we have:

$$
\begin{aligned}
\omega^{k+1} \circ \delta^{k}\left(\overrightarrow{\omega^{n}}\left(x_{1}, \ldots, x_{n}\right)\right) \\
\left.\quad=\sum \omega^{k+1} \overrightarrow{\omega^{i_{1}}}\left(x_{1}, \ldots, x_{i_{1}}\right), \ldots, \overrightarrow{\omega^{n-i_{k}}}\left(x_{i_{k}+1}, \ldots, x_{n}\right)\right) \\
\quad=\sum \omega^{k+2}\left(x_{1}, \overrightarrow{\omega^{i_{1}-1}}\left(x_{2}, \ldots, x_{i_{1}}\right), \ldots, \overrightarrow{\omega^{n-l_{k}}}\left(x_{i_{k}+1}, \ldots, x_{n}\right)\right) \\
\quad=\sum \omega^{k+2}\left(\overrightarrow{\omega^{1}}\left(x_{1}\right), \overrightarrow{\omega^{i_{1}-1}}\left(x_{2}, \ldots, x_{i_{1}}\right), \ldots, \overrightarrow{\omega^{n-i_{k}}}\left(x_{i_{k}+1}, \ldots, x_{n}\right)\right) .
\end{aligned}
$$

Each term $\left.\omega^{k+1} \overrightarrow{\left(\omega^{i_{1}}\right.}\left(x_{1}, \ldots, x_{i_{1}}\right), \ldots, \overrightarrow{\omega^{n-i_{k}}}\left(x_{i_{k}+1}, \ldots, x_{n}\right)\right)$ appears twice in $e\left(\overrightarrow{\omega^{n}}\left(x_{1}, \ldots, x_{n}\right)\right)$ : in $\omega^{k+1} \circ \delta^{k}\left(\overrightarrow{\omega^{n}}\left(x_{1}, \ldots, x_{n}\right)\right)$ with coefficient $(-1)^{k}$, and in $\omega^{k+2} \circ \delta^{k+1}\left(\overrightarrow{\omega^{n}}\left(x_{1}, \ldots, x_{n}\right)\right)$ with coefficient $(-1)^{k+1}$. Since $e\left(\overrightarrow{\omega^{n}}\left(x_{1}, \ldots, x_{n}\right)\right)$ is the sum of these terms, we get $e\left(\overrightarrow{\omega^{n}}\left(x_{1}, \ldots, x_{n}\right)\right)=0$.

(ii) Using the non-unital infinitesimal relation verified by $\delta$, it is immediate to check that, for elements $x_{1}, \ldots, x_{n}$ of $\overline{\mathcal{H}}$, we have the following equality:

$$
\begin{aligned}
\delta \circ \omega^{n}\left(x_{1}, \ldots, x_{n}\right)= & \sum_{i=1}^{n-1} \omega^{i}\left(x_{1}, \ldots, x_{i}\right) \otimes \omega^{n-i}\left(x_{i+1}, \ldots, x_{n}\right) \\
& +\sum_{j=1}^{n} \omega^{j}\left(x_{1}, \ldots, x_{j-1}, x_{j(1)}\right) \otimes \omega^{n-j+1}\left(x_{j(2)}, x_{j+1}, \ldots, x_{n}\right) .
\end{aligned}
$$

The coassociativity of $\delta$ and the formula above imply:

$$
\begin{aligned}
\delta \circ \omega^{n} \circ \delta^{n-1}(x)= & \sum_{i=1}^{n-1} \omega^{i}\left(x_{(1)}, \ldots, x_{(i)}\right) \otimes \omega^{n-i}\left(x_{(i+1)}, \ldots, x_{(n)}\right) \\
& +\sum_{i=1}^{n} \omega^{i}\left(x_{(1)}, \ldots, x_{(i)}\right) \otimes \omega^{n+1-i}\left(x_{(i+1)}, \ldots, x_{(n+1)}\right) .
\end{aligned}
$$

So, for $i \geq 1$, the term $\omega^{i}\left(x_{(1)}, \ldots, x_{(i)}\right) \otimes \omega^{n-i}\left(x_{(i+1)}, \ldots, x_{(n)}\right)$ appears twice in $\delta \circ e(x)$ : in $\delta \circ \omega^{n} \circ \delta^{n-1}(x)$ with coefficient $(-1)^{n-1}$, and in $\delta \circ \omega^{n-1} \circ \delta^{n-2}(x)$ with coefficient $(-1)^{n-2}$, which ends the proof.

(iii) The assertion follows directly from the definition of $e$ and the definition of $\delta^{n}$. 
Lemma 5.3 Given a connected As $s^{c}$-Mag-bialgebra $\mathcal{H}$, for any $x \in \overline{\mathcal{H}}$ one has the following equality:

$$
x=e(x)+\overrightarrow{\omega^{2}}\left((e \otimes e)\left(\delta^{1}(x)\right)\right)+\cdots+\overrightarrow{\omega^{n+1}}\left(e^{\otimes n+1}\left(\delta^{n}(x)\right)\right)+\cdots
$$

Proof Let $x \in F_{r} \mathcal{H}$, with $r \geq 1$. If $r=1$, then the result is obvious.

For $r>1$, note that $e(x)=x-e\left(x_{(1)}\right) \cdot x_{(2)}$, and that

$$
\overrightarrow{\omega^{n+1}}\left(e^{\otimes n+1}\left(\delta^{n}(x)\right)\right)=e\left(x_{(1)}\right) \cdot \overrightarrow{\omega^{n}}\left(e^{\otimes n}\left(\delta^{n-1}\left(x_{(2)}\right)\right) .\right.
$$

Since any $x_{(2)} \in F_{r-1} \mathcal{H}$, it verifies the equality, so one gets:

$$
\begin{aligned}
& \sum_{i \geq 0} \overrightarrow{\omega^{i+1}}\left(e^{\otimes i+1}\left(\delta^{i}(x)\right)\right) \\
& \quad=x-e\left(x_{(1)}\right) \cdot x_{(2)}+\sum_{i \geq 1} \overrightarrow{\omega^{i+1}}\left(e^{\otimes i+1}\left(\delta^{i}(x)\right)\right) \\
& \quad=x-e\left(x_{(1)}\right) \cdot x_{(2)}+\sum_{i \geq 1}\left(e\left(x_{(1)}\right) \cdot \vec{\omega}^{i}\left(e^{\otimes i}\left(\delta^{i-1}\left(x_{(2)}\right)\right)\right)\right. \\
& \quad=x-e\left(x_{(1)}\right) \cdot x_{(2)}+e\left(x_{(1)}\right) \cdot x_{(2)}=x .
\end{aligned}
$$

Let us now prove Theorem 4.1.

Proof (a) $\Rightarrow$ (c) Let $\mathcal{H}$ be a connected coassociative magmatic bialgebra, with coproduct $\Delta=i d_{\mathcal{H}} \otimes 1+1 \otimes i d_{\mathcal{H}}+\delta$. In the following we do not omit the summation sign, but indicate it in the notation $\delta(x)=\sum x_{(1)} \otimes x_{(2)}$. We note that the map

$$
x \mapsto \sum_{n \geq 1}\left(\sum e\left(x_{(1)}\right) \otimes \cdots \otimes e\left(x_{(n)}\right)\right) \in \bigoplus_{n}(\operatorname{Prim} \mathcal{H})^{\otimes n}
$$

gives a linear bijection $\alpha$ from $\overline{\mathcal{H}}$ into $\overline{T^{c}(\operatorname{Prim} \mathcal{H})}$. Moreover, we have $\delta^{c}\left(e\left(x_{(1)}\right) \otimes \cdots \otimes e\left(x_{(n)}\right)\right)=\sum_{i=1}^{n-1}\left(e\left(x_{(1)}\right) \otimes \cdots \otimes e\left(x_{(i)}\right)\right) \otimes\left(e\left(x_{(i+1)}\right) \otimes \cdots \otimes e\left(x_{(n)}\right)\right)$

for $n \geq 1$, where $\delta^{c}$ is the reduced deconcatenation coproduct on $\overline{T^{c}(\operatorname{Prim} \mathcal{H})}$. On the other hand, let $\delta^{i-1}\left(x_{(1)}\right)=\sum x_{(1,1)} \otimes \cdots \otimes x_{(1, i)}$ and $\delta^{n-i-1}\left(x_{(2)}\right)=\sum x_{(2, i+1)} \otimes$ $\cdots \otimes x_{(2, n)}$. The coassociativity of $\Delta$ implies that

$$
\delta^{n-1}(x)=\sum x_{(1)} \otimes \cdots \otimes x_{(n)}=\sum\left(\sum x_{(1,1)} \otimes \cdots \otimes x_{(2, n)}\right) .
$$


Then we have

$$
\begin{aligned}
& \sum \alpha\left(x_{(1)}\right) \otimes \alpha\left(x_{(2)}\right) \\
& =\sum\left(\sum_{i \geq 1} e\left(x_{(1,1)}\right) \otimes \cdots \otimes e\left(x_{(1, i)}\right)\right) \otimes\left(\sum_{n-i \geq 1} e\left(x_{(2, i+1)} \otimes \cdots \otimes e\left(x_{(2, n)}\right)\right)\right) \\
& =\sum_{n \geq 1}\left(\sum_{i=1}^{n-1}\left(\left(e\left(x_{(1)}\right) \otimes \cdots \otimes e\left(x_{(i)}\right)\right) \otimes\left(e\left(x_{(i+1)}\right) \otimes \cdots \otimes e\left(x_{(n)}\right)\right)\right)\right) \\
& =\delta^{c}(\alpha(x)),
\end{aligned}
$$

which implies that $\alpha$ is a coalgebra isomorphism.

(c) $\Rightarrow$ (b) We consider the coalgebra isomorphism $T^{c}(\operatorname{Prim} \mathcal{H}) \rightarrow U(\operatorname{Prim} \mathcal{H})$ which sends $x_{1} \otimes \cdots \otimes x_{n} \mapsto \overrightarrow{\omega^{n}}\left(x_{1}, \ldots, x_{n}\right)$. Since $\mathcal{H}$ is cofree, we obtain that $\mathcal{H}$ is isomorphic to $U(\operatorname{Prim} \mathcal{H})$ as a coalgebra. An easy calculation shows that the isomorphism is also an isomorphism of magmatic algebras.

(b) $\Rightarrow$ (a) is obvious.

The idempotents $e$ and $e^{v} \mathbf{5 . 4}$ The theory of generalized bialgebras gives an idempotent $e^{v}: \operatorname{Mag}(V) \rightarrow \operatorname{Mag}(V)$, called the versal idempotent, once a section $s: A s^{c} \rightarrow$ Mag has been chosen. In 4.2 we chose for $s$ the left comb product. In 5.1 we constructed and used an other idempotent $e$. In this paragraph we give the value of these two idempotents in low dimension, that is, we make explicit the maps $e_{n}^{v}$ and $e_{n}: \operatorname{Mag}_{n} \rightarrow \operatorname{Mag}_{n}$ for $n=2,3,4$.

\begin{tabular}{|c|c|c|c|c|c|c|}
\hline$t$ & $-\cdot-$ & $(-\cdot-) \cdot-$ & $-\cdot(-\cdot-)$ & & & \\
\hline$e(t)$ & 0 & $\mu_{1}^{3}$ & 0 & & & \\
\hline$e^{v}(t)$ & 0 & 0 & $-\mu_{1}^{3}$ & & & \\
\hline$t$ & $((-\cdots$ & $\cdot-) \cdot-$ & $(-\cdot(-\cdot-)) \cdot-$ & $(---) \cdot(-\cdot-)$ & $-\cdot((-\cdot-) \cdot-)$ & $-\cdot(-\cdot(-\cdot-))$ \\
\hline$e(t)$ & & $\iota_{1}^{4}$ & $\mu_{1}^{4}$ & $\mu_{2}^{4}$ & 0 & 0 \\
\hline$e^{v}(t)$ & & 0 & 0 & $\mu_{2}^{4}-\mu_{1}^{4}$ & $-\mu_{1}^{4}$ & $-\mu_{1}^{4}$ \\
\hline
\end{tabular}

Under the notation introduced 3.5 we have

$$
\begin{aligned}
& \mu_{1}^{3}=\text { as }:=((-\cdot) \cdot-)-(-\cdot(-\cdot)) \\
& \mu_{1}^{4}:=((-\cdot(-\cdot)) \cdot-)-(-\cdot((-\cdot-) \cdot-)), \\
& \mu_{2}^{4}:=-(((-\cdot) \cdot-) \cdot-)+((-\cdot(-\cdot-)) \cdot-)+((-\cdot) \cdot(-\cdot-))-(-\cdot(-\cdot(-\cdot-))) .
\end{aligned}
$$

The computation gives the following results:

Acknowledgements R. Holtkamp wants to thank the I.R.M.A. (Université Louis Pasteur, Strasbourg) for the hospitality. R. Holtkamp and J.-L. Loday thank the Mittag-Leffler Institute for an invitation in April 2004 during which this work was started. The authors thank Bruno Vallette for his remarks on the first version of this paper. 


\section{References}

1. Aguiar, M.: Infinitesimal Hopf algebras. In: New Trends in Hopf Algebra Theory, La Falda, 1999. Contemporary Mathematics, vol. 267, pp. 1-29. Am. Math. Soc., Providence (2000)

2. Bergeron, F., Labelle, G., Leroux, P.: Combinatorial Species and Tree-like Structures. Encyclopedia of Mathematics and its Applications, vol. 67. Cambridge University Press, Cambridge (1998) (xx+457 pp. Translated from the 1994 French original by M. Readdy, with a foreword by G.-C. Rota)

3. Burgunder, E.: Infinite magmatic bialgebras. Adv. Appl. Math. (2007, to appear)

4. Cartier, P.: Hyperalgèbres et groupes de Lie formels. Séminaire Sophus Lie, 2e année: 1955/56. Faculté des Sciences de Paris

5. Deutsch, E., Shapiro, L.: A survey of the Fine numbers. Selected papers in honor of Helge Tverberg. Discrete Math. 241(1-3), 241-265 (2001)

6. Ginzburg, V., Kapranov, M.: Koszul duality for operads. Duke Math. J. 76(1), 203-272 (1994)

7. Hofmann, K.H., Strambach, K.: Topological and analytic loops. In: Chein, O., et al. (eds.) Quasigroups and Loops: Theory and Applications, pp. 205-262. Heldermann, Berlin (1990)

8. Holtkamp, R.: Comparison of Hopf algebras on trees. Arch. Math. (Basel) 80(4), 368-383 (2003)

9. Holtkamp, R.: On Hopf algebra structures over free operads. Adv. Math. 207(2), 544-565 (2006)

10. Loday, J.-L.: Generalized bialgebras and triples of operads. Preprint (2006); ArXiv: math.QA/0611885

11. Loday, J.-L., Ronco, M.: Hopf algebra of the planar binary trees. Adv. Math. 139, 293-309 (1998)

12. Loday, J.-L., Ronco, M.: Algèbres de Hopf colibres. C. R. Math. Acad. Sci. Paris 337(3), 153-158 (2003)

13. Loday, J.-L., Ronco, M.: On the structure of cofree Hopf algebras. J. Reine Angew. Math. 592, 123155 (2006)

14. Milnor, J.W., Moore, J.C.: On the structure of Hopf algebras. Ann. Math. (2) 81, 211-264 (1965)

15. Markl, M., Shnider, S., Stasheff, J.: Operads in Algebra, Topology and Physics. Mathematical Surveys and Monographs, vol. 96. AMS, Providence (2002) (x+349 pp.)

16. Patras, F.: L'algèbre des descentes d'une bigèbre graduée. J. Algebra 170(2), 547-566 (1994)

17. Quillen, D.: Rational homotopy theory. Ann. Math. (2) 90, 205-295 (1969)

18. Reutenauer, C.: Theorem of Poincaré-Birkhoff-Witt, logarithm and symmetric group representations of degrees equal to Stirling numbers. In: Combinatoire Énumérative, Montreal, 1985. Lecture Notes in Mathematics, vol. 1234, pp. 267-284. Springer, New York (1986)

19. Ronco, M.: Eulerian idempotents and Milnor-Moore theorem for certain non-cocommutative Hopf algebras. J. Algebra 254(1), 152-172 (2002)

20. Shestakov, I.P., Umirbaev, U.U.: Free Akivis algebras, primitive elements, and hyperalgebras. J. Algebra 250(2), 533-548 (2002)

21. Solomon, L.: On the Poincaré-Birkhoff-Witt theorem. J. Comb. Theory 4, 363-375 (1968)

22. Vallette, B.: A Koszul duality for props. Trans. AMS 359, 4865-4943 (2007) 